\title{
PRODUÇÃO ANIMAL EM GRAMÍNEAS DE ESTAÇÃO FRIA COM FERTILIZAÇÃO NITROGENADA OU ASSOCIADAS COM LEGUMINOSA, COM OU SEM FERTILIZAÇÃO NITROGENADA ${ }^{1}$
}

\author{
ANIMAL PRODUCTION ON COOL SEASON GRASSES WITH NITROGEN FERTILIZATION \\ OR ASSOCIATED WITH LEGUME WITH OR WITHOUT NITROGEN FERTILIZATION
}

\author{
Manoel Flores Lesama ${ }^{2}$ Eduardo Londero Moojen ${ }^{3}$
}

RESUMO

O experimento foi conduzido em Santiago, $R S$, Brasil. Foram avaliados no período de 24/04/96 a 05/11/96, distintos sistemas de produção animal, nos quais os tratamentos constavam de uma pastagem de aveia preta (Avena strigosa, Schreb.) + azevém anual (Lolium multiflorum, Lam.) + trevo vesiculoso (Trifolium vesiculosum, Savi) $(\boldsymbol{A V}+\boldsymbol{A Z}+\boldsymbol{T} v)$; aveia preta + azevém anual + trevo vesiculoso $+150 \mathrm{~kg}$ de nitrogênio $(\boldsymbol{A} \boldsymbol{V}+$ $\boldsymbol{A Z}+\boldsymbol{T} \boldsymbol{v}+\boldsymbol{N})$ e aveia preta + azevém anual $+300 \mathrm{~kg}$ de nitroge $\hat{e}-$ nio $(\boldsymbol{A} \boldsymbol{V}+\boldsymbol{A Z}+\boldsymbol{N})$ num delineamento completamente casualizado, com três repetições. $O$ sistema de pastejo foi o contínuo, com lotação variável para manter a oferta de matéria seca (pressão de pastejo) em 10\%. Foram utilizados terneiros desmamados. Os parâmetros avaliados foram carga animal (CA), ganho médio diário $(G M D)$ e ganho de peso vivo por hectare. As CA foram maiores nos tratamentos com $N$. As cargas foram de $1140 ; 1490$ e $1652 \mathrm{~kg} P V /$ ha para os tratamentos $A V+A Z+T v ; A V+A Z+T v$ $+N$ e $A V+A Z+N$ respectivamente. Os GMD foram de 0,928; 1,091 e 0,839kg/an/dia, determinando ganhos de peso vivo por hectare de 515; 720 e 650kg/ha para os tratamentos $A V+A Z$ $+T v ; A V+A Z+T v+N e A V+A Z+N$, respectivamente. Os resultados obtidos sugerem a utilização da fertilização nitrogenada em pastagens de aveia preta + azevém anual + trevo vesiculoso, criando uma nova alternativa para aumentar a eficiência de produção e colher altos ganhos de peso vivo por área.

Palavras-chave: pastejo contínuo, fertilização nitrogenada, Trifolium vesiculosum, Lolium multiflorum, Avena strigosa.

\section{SUMMARY}

The experiment was conducted in Santiago, RS Brazil, during the period from April 24, 1996 to May 11, 1996.

\begin{abstract}
Animal production systems in pastures were evaluated in which the treatments were comparisons between oats + annual ryegrass + arrowleaf clover $(O+R+A)$; oats + annual ryegrass + arrowleaf clover $+150 \mathrm{~kg} / \mathrm{ha}$ of nitrogen $(O+R+A+N)$ and oats + annual ryegrass $+300 \mathrm{~kg} / \mathrm{ha}$ of nitrogen $(O+R+N)$, in a complete randomized design, with three replications. The grazing method was the continuous, with stock adjustements to maintain the dry matter on offer (grazing pressure) in 10\%. It was used weaned calves. The parameters evaluated were stocking rate, average daily gain per animal $(A D G)$ and liveweigth gain per hectare $(L W G)$. The stocking rates were higher in the treatments with nitrogen. They were 1140, 1490 and $1652 \mathrm{~kg} \mathrm{LW} / \mathrm{ha}$, respectively to $O+R+A ; O+R+A+N$ and $O+R+N$. The $A D G$ were 0.9281 .091 and $0.839 \mathrm{~kg} / \mathrm{an} /$ day, determining liveweight gains per hectare of 515; 720 and $650 \mathrm{~kg} \mathrm{LW/ha}$, to the treatments $O+R+A ; O+R+A+N$ and $O+R+N$. The obtained results suggest the utilization of nitrogen fertilization in pastures of oats + annual ryegrass + arrowleaf clover, raising a new alternative to increase the efficiency of pastures production, reduce costs, and achieve hight liveweight gains per area.
\end{abstract}

Key words: continuous grazing, nitrogen fertilizer, Trifolium vesiculosum, Lolium multiflorum, Avena strigosa.

\section{INTRODUÇÃO}

O nitrogênio é freqüentemente visto como limitante para a produção de biomassa nos sistemas de produção do Cone Sul. A pesquisa tem direcionado sua visão para aumentar a quantidade ou a eficiência de produção e, ao mesmo tempo, avaliar os impactos dos diferentes métodos e alternativas de intensificação de uso do solo. Isto tem levado a um

\footnotetext{
${ }^{1}$ Parte da Dissertação de Mestrado apresentada pelo primeiro autor para obtenção do grau de Mestre em Zootecnia na Universidade Federal de Santa Maria (UFSM), Santa Maria, RS.

${ }^{2}$ Engenheiro Agrônomo, Mestre, Bolsista da CAPES

${ }^{3}$ Engenheiro Agrônomo, Doutor, Professor Titular, Departamento de Zootecnia, UFSM, 97105-900, Santa Maria, RS. Autor para correspondência. E-mail: moojenel@ccr.ufsm.br
} 
aumento do interesse pelo uso de leguminosas, devido a seu baixo impacto, mas reduzindo o rendimento dos sistemas pastoris. Tais benefícios podem ser encontrados em ambos os sistemas, simplesmente pela redução, sobretudo da fertilização nitrogenada, e assim, possivelmente, da produtividade do sistema (PARSONS et al., 1991). Uma exceção é a Nova Zelândia (NZ) onde uma pastagem consorciada bem manejada, freqüentemente tem produções de forragem entre 10 - 15t de MS/ha/ano (DALY, 1990). A diferença entre a Grã-Bretanha (GB) e a NZ, as quais têm áreas similares de pastagens, é ilustrada pelo fato de que, aproximadamente, $1.000 .000 \mathrm{t}$ de fertilizante $\mathrm{N}$ é utilizado a cada ano nas pastagens da GB, mas unicamente 10.000 a $20.000 t$ de fertilizante $\mathrm{N}$ são utilizados na NZ. A fixação de $\mathrm{N}$ pelas leguminosas tem sido estimada como fornecendo cerca de 1.000.000t de N/ano na NZ, mas somente 80.000t é fixado na GB (WHITEHEAD, 1995). Na Argentina, a região Pampeana está semeada com aproximadamente 5.000.000 ha de alfafa (Medicago sativa), estimando-se uma fixação de 500.000t de N/ano. Nesta situação, esta leguminosa é a principal protagonista dos sistemas que produzem leite e carne, assim como sustenta a produção de cereais, os quais tem vantagens e oportunidades competitivas em nível mundial (PORDOMINGO, 1995).

A produção animal em pastagens de gramíneas de estação fria é dependente da relação entre o comportamento animal e os atributos das pastagens, estes últimos, principalmente influenciados pelo nível de fertilizante nitrogenado utilizado. O principal benefício da inclusão de leguminosas em pastagens de gramíneas de estação fria é devido ao fato que as leguminosas melhoram o valor da dieta apreendida pelo animal em pastejo (POPPI \& McLLENAN, 1995). Existe um histórico de trabalhos de pesquisa, envolvendo pastagens cultivadas de inverno sob pastejo, entre eles, o estudo conduzido por SCHOLL et al., (1976) durante a década de 70, com aveia branca (Avena sativa L.), utilizando uma dose de nitrogênio de $90 \mathrm{~kg}$ de N/ha. QUADROS \& MARASCHIN (1987), comparando três misturas, obtiveram para aveia, azevém e trevo vesiculoso (Trifolium vesiculosum) um ganho médio diário de $0,705 \mathrm{~kg}$ e um rendimento de $495 \mathrm{~kg}$ de peso vivo/ha, utilizando terneiros desmamados. Conhecendo estes resultados, MOOJEN (1993) começou a utilizar doses crescentes de nitrogênio em pastagens de milheto (Pennisetum americanum), obtendo ganhos de 245 , 455 e $655 \mathrm{~kg}$ de PV/ha, com produções de MS de 6689, 11339 e $15989 \mathrm{~kg} / \mathrm{ha}$, respectivamente, nas doses de 0,150 e $300 \mathrm{~kg} / \mathrm{ha}$ de N. Da mesma forma, RESTLE et al (1993) e LUPATINI et al. (1993) trabalharam em mistura de aveia + azevém, com novilhos, utilizando três níveis de nitrogênio $(0,150$ e $300 \mathrm{~kg}$ de $\mathrm{N} / \mathrm{ha})$, colhendo 335,641 e $865 \mathrm{~kg}$ de $\mathrm{PV} / \mathrm{ha}$, e as produções de MS foram de 4893, 9327 e 10905kg/há, mostrando assim a excelente resposta destas gramíneas de inverno quando bem adubadas e submetidas a um bom manejo de pastejo.

O crescimento da gramínea é aumentado pela fertilização $\mathrm{N}$, e como conseqüência, a quantidade de leguminosas na pastagem é freqüentemente reduzida (DAVIS \& EVANS, 1990). A redução no crescimento da leguminosa é devido principalmente ao aumento da competição por luz, água e/ou nutrientes (FRAME \& NEWBOULD, 1986). A leguminosa cresce bem unicamente, quando o fornecimento de $\mathrm{N}$ é insuficiente para um máximo crescimento das gramíneas (HARRIS, 1990). Em muitos lugares, há uma discussão com relação ao mérito da leguminosa e/ou do fertilizante N, e em qual extensão será possível combinar as duas fontes numa mesma vegetação. Nos EUA, BURNS \& STANDAERT (1984) estimaram que são necessários $200 \mathrm{~kg}$ de N/ha para pastagens de gramíneas puras alcançarem produções similares a das pastagens consorciadas. Já na NZ, há indicações que são necessários de 300 a $600 \mathrm{~kg}$ de $\mathrm{N} / \mathrm{ha}$ para que as pastagens de gramíneas puras possam manter o mesmo nível de produção de forragem atingido por diferentes pastagens consorciadas bem manejadas, sustentando constantemente uma fixação anual entre 150 a $300 \mathrm{~kg}$ de N/ha (BALL \& FIELD, 1984). Na GB, dados mostram que maiores produções são obtidas com pastagens consorciadas fertilizadas com $\mathrm{N}$, do que com pastagens de gramíneas em monocultura fertilizadas com $\mathrm{N}$ em taxas pelo menos, acima de $300 \mathrm{~kg} / \mathrm{ha} / \mathrm{ano}$, e isso sugere que pode ser econômico o uso de leguminosas nas pastagens, recebendo taxas moderadas de fertilizante $\mathrm{N}$ (WHITEHEAD, 1995). O uso de pastagens consorciadas leguminosa-gramínea em combinação com algum fertilizante $\mathrm{N}$ tem sido recomendado por vários autores (WHITEHEAD, 1995).

\section{MATERIAL E MÉTODOS:}

O experimento foi conduzido na Fazenda Santiago, pertencente ao Grupo Nicola, no município de Santiago (RS). Os tratamentos constaram da seguinte comparação entre misturas forrageiras de gramíneas com fertilização nitrogenada e/ou leguminosa: $\mathrm{AV}+\mathrm{AZ}+\mathrm{Tv}$ - aveia preta (Avena strigosa), azevém (Lolium multiflorum) e trevo vesiculoso cv. Yuchi (Trifolium vesiculosum); AV + AZ + $\mathrm{Tv}+\mathrm{N}$ - aveia preta, azevém, trevo vesiculoso e $150 \mathrm{~kg}$ de nitrogênio; $\mathrm{AV}+\mathrm{AZ}+\mathrm{N}$ - aveia preta, azevém e $300 \mathrm{~kg}$ de nitrogênio.

Segundo MORENO (1961), o clima da região é do tipo Cfa, subtropical úmido, com verão 
quente. O solo é classificado como Latossolo Vermelho - Escuro Álico (IBGE, 1986). O período de utilização compreendeu 96 dias (02/08 e 05/11/96). O delineamento experimental foi o completamente casualizado com três repetições, totalizando 8,4 hectares, divididos em potreiros de 1,0 ha para os tratamentos com leguminosas e 0,8 para os demais. O preparo foi executado de 17 a 21/04/96. A adubação de base foi realizada em 24/04/96, segundo a recomendação de SIQUEIRA et al. (1989); constou da aplicação de $3700 \mathrm{~kg} / \mathrm{ha}$ de calcário e $400 \mathrm{~kg} / \mathrm{ha}$ da fórmula 05-25-25. O fertilizante nitrogenado utilizado foi o nitrato de amônio $(34 \% \mathrm{~N})$, aplicado em cobertura, sendo distribuído em três oportunidades. Os animais experimentais utilizados foram novilhos cruzas, Blond-Caracu e Braford desmamados em maio, que entraram no experimento com idade entre 9 e 10 meses. Os animais entraram nos potreiros em 06/08/96, ou seja, 72 dias após a semeadura. O método de pastejo empregado foi o contínuo e o nível de oferta de forragem por hectare foi $10 \mathrm{~kg}$ $\mathrm{MS} / 100 \mathrm{~kg}$ de peso vivo/dia. Usou-se a técnica das lotações variáveis "put-and-take", descrita por MOTT \& LUCAS (1952), para manter os níveis de oferta de forragem pretendidos, com 4 animais "testers" por potreiro. Para o cálculo da carga animal por potreiro, utilizou-se a matéria seca disponível, que constava da MS amostrada pela realização dos cortes, acrescida da taxa de acumulação diária estimada no período anterior e projetada para o seguinte pe-ríodo e dividida pelo número de dias transcorridos. $\mathrm{O}$ ganho médio diário dos animais "testers" foi obtido pela diferença entre pesagens realizadas no início e final de cada período experimental. Determinou-se o ganho de peso por hectare, multiplicando-se o ganho médio diário dos animais "testers" pelo número de animais dia/ha. As análises de ganho de peso médio diário, carga animal e ganho de peso vivo por hectare foram determinadas através da análise da variância e teste da Probabilidade da diferença (Ppdiff), utilizando diferentes níveis de significância pelo teste $\mathrm{T}$ de Student.

\section{RESULTADOS E DISCUSSÃO}

Os valores observados para carga animal expressa emkg PV/ha/dia estão presentes na Tabela 1. Na média ponderada entre os tratamentos, observou-se o maior suporte dos tratamentos adubados com $\mathrm{N}$, em vista das altas taxas diárias de acúmulo, permitindo alcançar alta produção de matéria seca nestes tratamentos, mostrando a importância deste nutriente nos sistemas de produção de carne. A resposta obtida na média ponderada, mostrou um aumento de $2,3 \mathrm{~kg}$ de $\mathrm{PV} / \mathrm{ha}$ para cada $\mathrm{kg}$ de $\mathrm{N}$ aplicado, para os tratamentos onde a contribuição da leguminosa é importante para o ganho por animal, evidenciando um potencial de produção de ganho de peso vivo por área.

A oferta calculada resultou nas cargas animais utilizadas, assim ao longo do período a carga evoluiu da seguinte forma: inicialmente, o tratamento $\mathrm{AV}+\mathrm{AZ}+\mathrm{N}$ suportou uma maior carga $(\mathrm{P}<0,0215)$, seguido do $\mathrm{AV}+\mathrm{AZ}+\mathrm{Tv}+\mathrm{N}$ e $\mathrm{AV}+$ $\mathrm{AZ}+\mathrm{Tv}$ que não diferiram entre si. A seguir o $\mathrm{AV}+$ $\mathrm{AZ}+\mathrm{N}$ continuou tendo maior carga, apresentando uma diferença significativa $(\mathrm{P}<0,0026)$ em relação aos demais tratamentos; este fato ocorreu devido à alta taxa de acúmulo de forragem deste período,

Tabela 1 - Carga animal em kg de peso vivo por hectare nos períodos e na média ponderada, de gramíneas de estação fria com fertilização nitrogenada ou associadas com leguminosa, com ou sem fertilização nitrogenada. Fazenda Santiago, Santiago, RS.

\begin{tabular}{|c|c|c|c|c|c|}
\hline & \multicolumn{4}{|c|}{ DATAS } & \multirow[b]{2}{*}{ Média Ponderada } \\
\hline & $06 / 08$ & 04/09 & $\begin{array}{l}08 / 10 \\
(\mathrm{Kg} P \mathrm{P}\end{array}$ & $\begin{array}{l}05 / 11 \\
\text { a ) }\end{array}$ & \\
\hline$A V+A Z+T v^{1}$ & $779^{b *}$ & $1297^{\mathrm{c}}$ & $1307^{\mathrm{b}}$ & $964^{c}$ & $1140^{\mathrm{b}}$ \\
\hline$A V+A Z+T v+N^{2}$ & $956^{\mathrm{b}}$ & $1582^{b}$ & $1912^{a}$ & $1810^{a}$ & $1490^{\mathrm{a}}$ \\
\hline$A V+A Z+N^{3}$ & $1179^{\mathrm{a}}$ & $1881^{\mathrm{a}}$ & $1874^{\mathrm{a}}$ & $1160^{\mathrm{b}}$ & $1652^{\mathrm{a}}$ \\
\hline média & 978,2 & 1587,2 & 1697,7 & 1311,5 & 1427,66 \\
\hline F & 7,79 & 18,78 & 9,64 & 103,59 & 11,31 \\
\hline $\mathrm{P}<$ & 0,0215 & 0,0026 & 0,0134 & 0,0001 & 0,0092 \\
\hline $\begin{array}{l}\text { * Médias seguidas da } \\
{ }^{1} \text { Aveia + azevém+ tr } \\
{ }^{2} \text { Aveia + azevém + tr } \\
{ }^{3} \text { Aveia + azevém + } 3\end{array}$ & $\begin{array}{l}\text { esma letra n } \\
\text { o vesiculos } \\
\text { vo vesiculos } \\
\mathrm{kg} / \mathrm{ha} \text { de ni }\end{array}$ & $\begin{array}{l}\text { coluna não } \\
+150 \mathrm{~kg} / \mathrm{h} \\
\text { ogênio. }\end{array}$ & $\begin{array}{l}\text { diferem esta } \\
\text { de nitrogên }\end{array}$ & ticamente e & tre si. \\
\hline
\end{tabular}

Ciência Rural, v. 29, n. 1, 1999. 
associada à alta adubação fertilizada. A carga foi novamente ajustada no dia $08 / 10$, com o objetivo de ajustar os resíduos, onde apenas o $\mathrm{AV}+\mathrm{AZ}+\mathrm{Tv}$ apresentou diferença significativa $(\mathrm{P}<0,0134)$, sendo inferior aos dois outros. A última avaliação da carga animal mostrou diferença $(\mathrm{P}<0,0001)$ entre os tratamentos, onde o $\mathrm{AV}+\mathrm{AZ}+\mathrm{TV}+\mathrm{N}$ desta vez suportou a maior carga, o $\mathrm{AV}+\mathrm{AZ}+\mathrm{N}$ foi intermediário e o $\mathrm{AV}+\mathrm{AZ}+\mathrm{TV}$ inferior.

$\mathrm{O}$ ganho médio diário por animal (kg/animal/dia), nos diferentes tratamentos, é apresentado na Tabela 2. Observando as médias dos GMD notou-se que o AV + AZ + Tv + N foi superior aos demais tratamentos $(\mathrm{P}<0,0002)$. $\mathrm{O}$ maior ganho por animal obtido no tratamento $\mathrm{AV}+\mathrm{AZ}+$ $\mathrm{Tv}+\mathrm{N}$ deveu-se a maior qualidade da forragem em oferta. Outro ponto importante foi que os tratamentos $\mathrm{AV}+\mathrm{AZ}+\mathrm{Tv}$ e $\mathrm{AV}+\mathrm{AZ}+\mathrm{N}$ não apresentaram diferença significativa entre si. Como a disponibilidade de forragem não variou entre os tratamentos, os valores obtidos sugerem uma adequação com ALLDEN \& WHITTAKER (1970), pois estes afirmam que, com resíduos acima de $1800 \mathrm{~kg} / \mathrm{ha}$ de MS, o tempo de pastejo, associado à taxa de consumo, mantém-se estável, logo, a estrutura da pastagem determina os efeitos sobre o consumo de forragem em pastejo.

Avaliando o primeiro período, foi possível observar um bom ganho de peso nos três trata-

Tabela 2 - Ganho de peso médio diário em kg PV/an/dia nos períodos e na média, em gramíneas de estação fria com fertilização nitrogenada ou associadas com leguminosa, com ou sem fertilização nitrogenada. Fazenda Santiago, Santiago, RS

\begin{tabular}{lcccc}
\hline & $06 / 08$ a $04 / 09$ & $04 / 08$ a $08 / 10$ & $08 / 10$ a $05 / 11$ & média \\
& \multicolumn{4}{c}{$(\mathrm{Kg} \mathrm{PV} / \mathrm{an} / \mathrm{dia})$} \\
\cline { 2 - 5 } $\mathrm{AV}+\mathrm{AZ}+\mathrm{Tv}^{1}$ & $1,167^{\mathrm{a}^{*}}$ & $0,924^{\mathrm{b}}$ & $0,655^{\mathrm{a}}$ & $0,928^{\mathrm{b}}$ \\
$\mathrm{AV}+\mathrm{AZ}+\mathrm{Tv}+\mathrm{N}^{2}$ & $1,339^{\mathrm{a}}$ & $1,220^{\mathrm{a}}$ & $0,667^{\mathrm{a}}$ & $1,091^{\mathrm{a}}$ \\
$\mathrm{AV}+\mathrm{AZ}+\mathrm{N}^{3}$ & $1,069^{\mathrm{a}}$ & $0,918^{\mathrm{b}}$ & $0,435^{\mathrm{b}}$ & $0,839^{\mathrm{b}}$ \\
& & & \\
\hline & 1,182 & 1,003 & 0,560 & 0,955 \\
média & 34,6 & 17,9 & 40,9 & 40,81 \\
$\mathrm{C} . \mathrm{V}$. & 0,1428 & 0,0067 & 0,0007 & 0,0002 \\
$\mathrm{P}<$ & & & \\
\hline
\end{tabular}

* Médias seguidas da mesma letra na coluna não diferem estatisticamente entre si.

Aveia + azevém + trevo vesiculoso

Aveia + azevém + trevo vesiculoso $+150 \mathrm{~kg} / \mathrm{ha}$ de nitrogênio

Aveia + azevém $+300 \mathrm{~kg} / \mathrm{ha}$ de nitrogênio mentos, não apresentando diferenças estatísticas entre si $(\mathrm{P}<0,1428)$, mas foi notada uma leve inferioridade do tratamento onde havia somente gramíneas, quando comparado aos tratamentos que apresentavam trevos na composição botânica da pastagem. No entanto, ao entrar no segundo período, a modificação do resíduo e, conseqüentemente, da estrutura da pastagem determinaram mudanças no GMD, destacando-se o tratamento $\mathrm{AV}+\mathrm{AZ}+\mathrm{TV}+$ $\mathrm{N}$, que superou os demais tratamentos. É importante, neste caso, levar em conta o efeito conjunto da quantidade de forragem verde disponível e sua digestibilidade. Ao observar-se a terceira avaliação, foi encontrada uma queda abrupta do GMD em todos os tratamentos, sendo a maior queda no tratamento constituído apenas de gramíneas, seguido do tratamento que incluía gramíneas, trevo e fertilização nitrogenada. $\mathrm{O}$ tratamento onde foram utilizadas gramíneas e trevos sem fertilização nitrogenada manteve um GMD mais estável.

Para o ganho de peso vivo por hectare, as médias para os tratamentos, nos três períodos considerados e período total de avaliação são apresentadas na Tabela 3. Observando-se o período total, as diferenças entre os ganhos de peso vivo por hectare não foram significativas entre os tratamentos $\mathrm{AV}+$ $\mathrm{AZ}+\mathrm{Tv}+\mathrm{N}$ e $\mathrm{AV}+\mathrm{AZ}+\mathrm{N}$, que foram superiores ao $\mathrm{AV}+\mathrm{AZ}+\mathrm{TV}(\mathrm{P}<0,0034)$. Evidencia-se então, que as diferenças entre as pastagens, quanto à produção em produto animal, são reflexo, provavelmente no primeiro momento, da quantidade de MS produzida e depois da qualidade desta MS. Quando compara-se o tratamento AV + AZ + $\mathrm{Tv}$ com o tratamento $\mathrm{AV}+\mathrm{AZ}+\mathrm{TV}+$ $\mathrm{N}$, a produção de produto animal foi $39,6 \%$ superior para o tratamento com aplicação de fertilizante $\mathrm{N}$. Isso demonstra uma resposta média de 1,360 g de ganho por $\mathrm{kg}$ de $\mathrm{N}$ aplicado, muito semelhante aos valores encontrados por WHITEHEAD (1995) e BURNS \& STANDAERT (1984), para eficiência de produção de carne por $\mathrm{kg}$ de $\mathrm{N}$ aplicado. A eficiência de transformação média de $\mathrm{kg}$ de MS em kg de ganho de peso vivo para os tratamentos $\mathrm{AV}+\mathrm{AZ}$ $+\mathrm{Tv} ; \mathrm{AV}+\mathrm{AZ}+\mathrm{Tv}+\mathrm{N}$ e AV + AZ + $\mathrm{N}$ foram respectivamente de 12,$3 ; 11,6$ e 14,9kg de MS para cada $\mathrm{Kg}$ de ganho de peso vivo, sucessivamente.

Analisando por períodos, pode ser observado que no primeiro período houve uma diferença estatística entre os tratamentos com adubação 
Tabela 3 - Ganho de peso vivo por área, em kg/ha por período, e ganho de peso vivo total em gramíneas de estação fria com fertilização nitrogenada ou associadas com leguminosa, com ou sem fertilização nitrogenada. Fazenda Santiago, Santiago, RS.

06/08 a 04/09 05/08 a 08/10 09/10 a 05/11 Ganho total

$(\mathrm{Kg} / \mathrm{ha})$

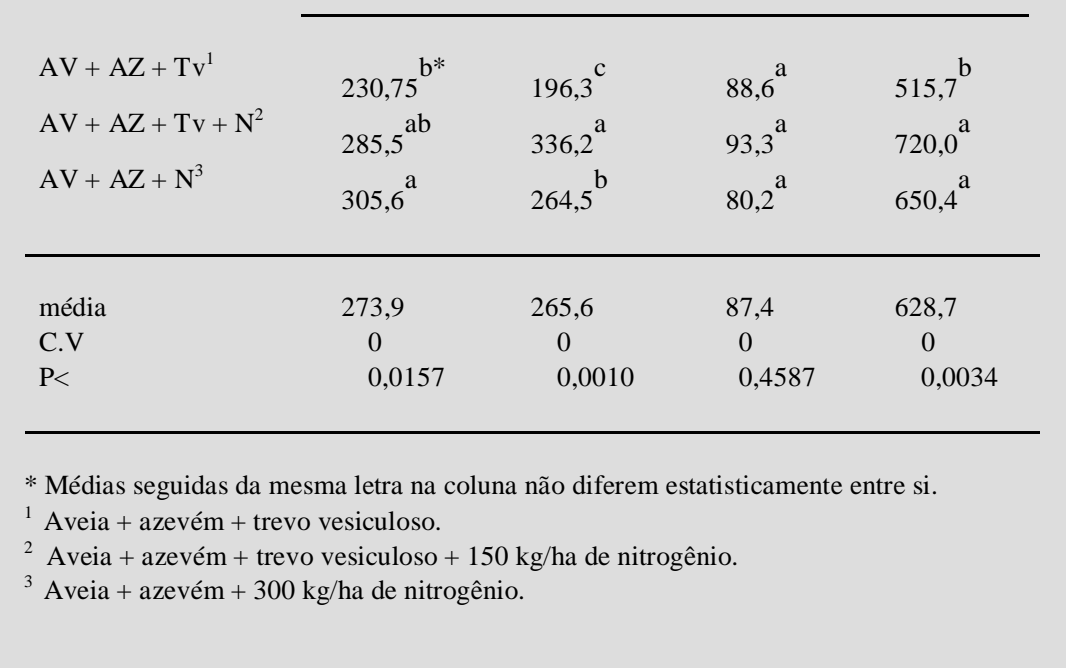

nitrogenada e o não adubado $(\mathrm{P}<0,0157)$. No segundo período, o tratamento $\mathrm{AV}+\mathrm{AZ}+\mathrm{Tv}+\mathrm{N}$ diferiu estatisticamente dos demais, destacando-se neste momento a sua qualidade no GMD e como este manteve uma carga alta, justificou sua superioridade. Quando se entra no terceiro período, as altas cargas utilizadas promoveram uma redução dos resíduos das pastagens e, a partir disto, uma redução do crescimento animal, penalizando drasticamente a digestibilidade da dieta neste período. Este fato vai ao encontro de HODGSON (1981), que afirma que a produção não varia muito, logo não é o fator determinante na diminuição do consumo e do desempenho animal com o acréscimo na lotação, e, segundo THOMPSON et al., (1994) e PROFFIT et al., (1993), é interessante intensificar a desfolhação das pastagens mediterrâneas no final do seu ciclo de crescimento anual, devido a vantagens na taxa de acúmulo das pastagens e na redução de seu valor nutritivo.

Observa-se que foram necessárias menores quantidades de MS a serem convertidas em produto animal nos tratamentos com a presença do componente trevo vesiculoso. Vê-se, então, que o ganho de peso vivo por hectare reflete a produção das pastagens, como também sua qualidade, quando considera-se uma mesma ou diferentes eficiências de transformação.

\section{CONCLUSÕES}

A utilização de $150 \mathrm{~kg}$ de nitrogênio em uma pastagem aveia + azevém + trevo vesiculoso pode substituir a aplicação de $300 \mathrm{~kg}$ de nitrogênio em uma pastagem de aveia + azevém, melhorando o ganho/animal e ganho/área. Torna-se necessário reavaliar as recomendações de fertilização nitrogenada preconizadas pela ROLAS, para pastagens consorciadas, já que este estudo confirma dados bibliográficos mundiais colhidos em condições de pastejo, os quais avaliam os retornos em produto animal, quantificando respostas positivas das pastagens consorciadas à aplicação de fertilizante nitrogenado.

\section{AGRADECIMENTOS}

Ao professor José Henrique Souza da Silva, do Departamento de Zootecnia da UFSM, pela orientação nas análises estatísticas deste trabalho.

\section{REFERÊNCIAS BIBLIOGRÁFICAS}

ALLDEN, W.G., WHITTAKER, I.A. The determinants of herbage intake by grazing sheep: the interrelationship of factors influencing herbage intake and availability. Australian Journal of Agricultural Research, Melbourne, v. 21 , n. 5 , p. $755-766,1970$.

BALL, P.R., FIELD, T.R.O. Productivity and economics of legume-based pastures and grass swards receiving fertiliser nitrogen in New Zealand. In: BARNES, R.F., BALL, P.R., BROUGHAM, R.W. et al. . Forage Legumes for EnergyEfficient Animal Production. Proceedings of a trilateral workshop held in Palmersthon North, 1984. New Zealand, April 30-May 4, 1984, p. 47-55.

BURNS, J.C., STANDAERT, J.E Productivity and economics of legume-based vs. Nitrogen-fertilized grass-based pastures in the United States. In: BARNES, R., BALL, R., BROUGHAM, R., et al. Forage legumes for energyefficient animal production. Proceedings of a trilateral workshop held in Palmersthon North, 1984. New Zealand, april 30-may 4, 1984, p. 56-71.

DALY, G.T. The grassland of New Zealand. In: Langer, R.H.M. Pastures, Their Ecology and Management. Auckland: Oxford University Press, 1990. Chap 1, p. 1-38.

DAVIS, A., EVANS, M.E. Effects of spring defoliation and fertilizer nitrogen on the growth of white clover in ryegrass/clover swards. Grass and Forage Science, Oxford, v. 45 , p. $345-356,1990$. 
FRAME, J.; NEWBOULD, P. Agronomy of white clover. Advances in Agronomy, 40, p. 1-88, p. 237-247, 1986.

HARRIS, W. Pasture as an ecosystem. In: LANGER. R.H.M (Ed.) Pastures: their ecology and management. Auckland: Oxford University Press, 1990. Chap.3, p. 75-131.

HODGSON, J. Variations in the surface characteristics of the sward and the short-term rate of herbage intake by calves and lambs. Grass and Forage Science, Oxford, 36, p. 49-57, 1981.

IBGE. FUNDAÇÃO INSTITUTO BRASILEIRO DE GEOGRAFIA E ESTATÍSTICA. Levantamento de recursos naturais. Rio de Janeiro, v. 33, Cap.2, p. 796. 1986.

LUPATINI, G.C., RESTLE, J.,CERETTA. $\boldsymbol{e}$ t $\boldsymbol{a l}$.. Avaliação da mistura de aveia (Avena strigosa L.) e azevém (Lolium multiflorum Lam.) sob pastejo submetida a níveis de nitrogênio. II- Produção de forragem. In: REUNIÃO ANUAL DA SOCIEDADE BRASILEIRA DE ZOOTECNIA, 30. 1993, Rio de Janeiro.Anais... Rio de Janeiro : Sociedade Brasileira de Zootecnia, 1993, 612 p., p.72.

MOOJEN, E. L. Avaliação do milheto (Pennisetum americanum L.) sob pastejo com níveis de nitrogênio. Santa Maria-RS. 39p. Tese (Progressão a Professor Titular) Departamento de Zootecnia, Universidade Federal de Santa Maria, 1993.

MORENO, J.A. Clima do Rio Grande do Sul. Porto Alegre, Secretaria da Agricultura, 1961, p. 41.

MOTT, G.O., LUCAS, H.L. The design, conduct and interpretation of grazing trials on cultivated and inproved pastures. In: International Grassland Congress, 6., 1952 Pennsylvania. Procedings... Pennsylvania: State College press, 1952. p. 1380-1385

PARSONS, A.J.; ORR, R.J.; PENNING, P.D.; LOCKYER, D.R. Uptake, cycling and fate of nitrogen in grass/clover swards continuously grazed by sheep. Journal of Agricultural Science, Cambridge, v. 16, p. 47-61. 1991.

POPPI, D.P.; McLLENAN, S.R. Protein and energy utilization by ruminants at pasture. Journal of Animal Science.
Champaign, v. 73, p. 278-290, 1995.

PORDOMINGO, A.J. Consideraciones económicas sobre a alfalfa. In: HIJANO, E.H.; NAVARRO, A. La alfafa en la Argentina. Publicación del Subprograma Alfalfa INTA C.R. Cuyo: 1995 v. 1, Cap. 13, p. 241-256.

PROFFIT, A.; BENDOTTI, S.; HOWELL, M. et al. The effect of sheep trampling and grazing on soil physical properties and pasture growth for a red-brown earth. Autralian Journal of Agricultural Research, Melbourne, v. 44, p. 317-331, 1993.

QUADROS, F.L.P., MARASCHIN, G.E. Desempenho animal em misturas de espécies forrageiras de estação fria. Pesquisa Agropecuária Brasileira, Brasília, v. 22, n. 5, p. 535-541, 1987.

RESTLE, J.; LUPATINI, G.C.; VALENTE, A.V. Produtividade animal em mistura de aveia preta (Avena strigosa L.) + azevém (Lolium multiflorum Lam.) sob pastejo submetidas a níveis de nitrogênio. I-Produção animal. In: REUNIÃO ANUAL DA SOCIEDADE BRASILEIRA DE ZOOTECNIA, 30., 1993, Rio de Janeiro. Anais... Rio de Janeiro: Sociedade Brasileira de Zootecnia, 1993, 612 p., p. 71.

SIQUEIRA,O.J.F.; SCHERER, E.R.; TASSINARI, G. $\boldsymbol{e}$ t al. Recomendações de adubação e calagem para os estados do Rio Grande do Sul e Santa Catarina. Passo Fundo: CNPT- Embrapa, 1989, 100 p.

SCHOLL, J.M.; LOBATO, J.F.P.; BARRETO, I.L. Improvement of pastures by direct seeding into native grass in southern Brazil with oats and with nitrogen supplied by fertilizer or arrowleaf clover. Turrialba, San José, 26, n. 2, p. 141-149, 1976.

THOMPSON, A.N.; DOYLE, P.T; GRIMM, M. Effects of stocking rate on liveweight and wool production of sheep grazing annual pastures. Australian Journal of Agricultural Research, Melbourne, v. 45, p. 367-389, 1994.

WHITEHEAD, D.C. Grassland Nitrogen., ed. Wallinford: CAB International. 1995, p. 397.

Ciência Rural, v. 29, n. 1, 1999. 